\title{
SPICE Compatible Equivalent Circuit Models of Skeletal Muscle Tissue
}

\author{
Robert Szlavik and Hubert De Bruin \\ Department of Electrical and Computer Engineering and Medicine \\ McMaster University, Hamilton, Ontario, Canada, L8N 3Z5
}

\begin{abstract}
We present equivalent circuit models of skeletal muscle tissue for the directions parallel and transverse to the fiber. The parallel model is based on a distributed parameter equivalent circuit approach where the tissue between the electrodes is divided into small segments, each of which is represented by an equivalent circuit. Parameters for the equivalent circuits are calculated from the electrical characteristics of the tissue components and their physical arrangement. We compare the conductivity frequency response of the equivalent circuit modelling approach to other models presented in the literature. The advantage to our equivalent circuit approach is that the tissue models can be readily integrated in circuit simulators such as SPICE. An equivalent circuit model of the tissue load that incorporates both spatial and frequency dependence can be simulated in combination with instrumentation circuits and can aid in the design and optimization of these systems. The possibility of using the equivalent circuit models to simulate nonlinear tissue characteristics is currently being investigated.
\end{abstract}

\section{Introduction}

Several authors have proposed models for the conductivity properties of tissue. Nicholson presented an analytical model for nerve fiber impedance in the direction transverse to the fibers [1]. More recently, Gielen et al. [2] applied Nicholson's model to skeletal muscle tissue and also presented an analytical model of the conductivity in the direction parallel to the muscle fibers. An alternate analytical model has been proposed by Plonsey et al. [3].

Our tissue equivalent circuit models expand on the analytical modelling of Nicholson and Gielen. The longitudinal equivalent circuit model is based on dividing the tissue into a finite number of segments. Each segment is represented by an equivalent circuit that is derived from the physical arrangement of the tissue components and their respective electrical parameters. Equivalent circuit models of the individual segments are then combined in series thus incorporating spatial dependence in the model.

\section{Method}

The physical basis for the longitudinal tissue conductivity equivalent circuit model is shown in Fig. 1. As in Gielen's [2] presentation, we approximate the muscle fiber as a hexagonal cylinder and assume the electronic and geometric parameters shown in Table 1. From these parameters and the geometry shown, the component values of the equivalent circuit model can be obtained.

\begin{tabular}{|c|c|c|c|}
\hline$\sigma_{i}$ & $5.000 \times 10^{-1} \mathrm{~S} / \mathrm{m}$ & $p$ & 0.9 \\
\hline$\sigma_{.}$ & $2.400 \mathrm{~S} / \mathrm{m}$ & $X$ & $5.000 \times 10^{-5} \mathrm{~m}$ \\
\hline$G_{m}$ & $3.333 \mathrm{~S} / \mathrm{m}^{2}$ & $Y$ & $4.333 \times 10^{-5} \mathrm{~m}$ \\
\hline$C_{m}$ & $2.500 \times 10^{-2} \mathrm{~F} / \mathrm{m}^{2}$ & $L$ & $2.400 \times 10^{-3} \mathrm{~m}$ \\
\hline$A$ & $5.000 \times 10^{-5} \mathrm{~m}$ & $d$ & $2.406 \times 10^{-6} \mathrm{~m}$ \\
\hline
\end{tabular}

Table 1. Electronic and geometric parameters for the tissue equivalent circuit models.

In Table $1, X, L$, and $Y$ are as shown in Fig. 1 and $A$ is the fiber intracellular radius which is equal to $X$. The ratio of the intracellular volume to the volume of the fiber is represented by $p$. Electrical parameters $G_{m}$ and $C_{m}$ are the membrane conductance and capacitance per unit area and $\sigma_{1}$ and $\sigma_{0}$ are the conductivities of the cytoplasmic and extracellular material. Calculated circuit parameter values are presented in Table 2. 


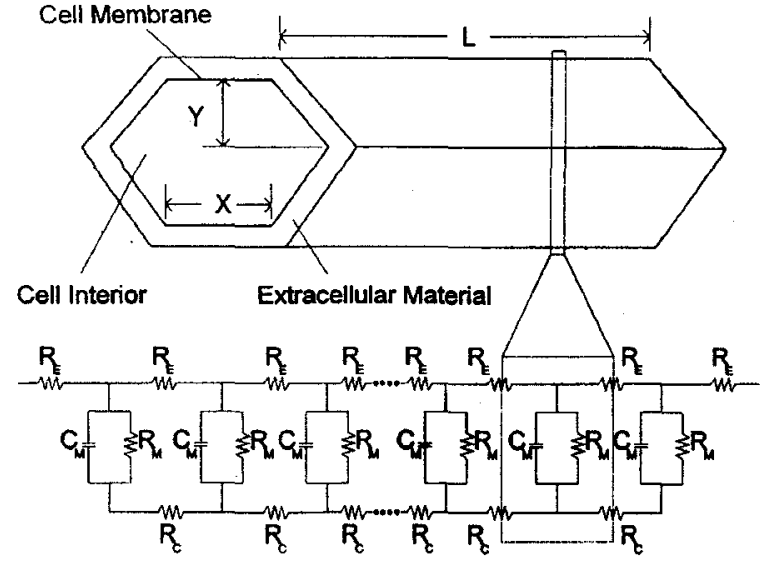

Figure 1. Conceptual and equivalent circuit model of tissue conductivity in the dinection parallel to the muscle fibers. The portion of the equivalent circuit outlined by the box outlines the equivalent circuit model for a single segment of the length of the fiber. Two dotted lines that join the circuit in the middle indicate that the circuit can be extended indefinitely to simulate longer inter-electrode spacings.

The circuits of Fig. 1. that are connected to one another in series represent a short segment of the fiber and the surrounding extracellular medium. For each short segment of the fiber, the current has an ohmic path through the extracellular medium $R_{E}$, an ohmic path through the intercellular material modelled by the resistance $R_{c}$ and a path across the cellular membrane represented by the parallel resistance and capacitance $R_{M}$ and $C_{M}$. This approach is analogous to Gielen's [2] model for an elemental length of fiber in the longitudinal direction. The fundamental difference between this modelling approach and that of Gielen's is that the approximated segments are of a finite length.

\begin{tabular}{|l|l|}
\hline$R_{E}$ & $5.771 \times 10^{4} \mathrm{D}$ \\
\hline$R_{C}$ & $3.079 \times 10^{4} \mathrm{0}$ \\
\hline$R_{M}$ & $1.000 \times 10^{7} \mathrm{Q}$ \\
\hline$C_{M}$ & $7.500 \times 10^{-10} \mathrm{~F}$ \\
\hline
\end{tabular}

Table 2. Circuit parameters for the longitudinal tissue equivalent circuit model.

The physical basis for the transverse equivalent circuit model is shown in Fig. 2a. As in Gielen's [2] model, we obtain the equivalent circuit for the transverse direction from the geometry of the cross section through the fibers that is perpendicular to the longitudinal direction.

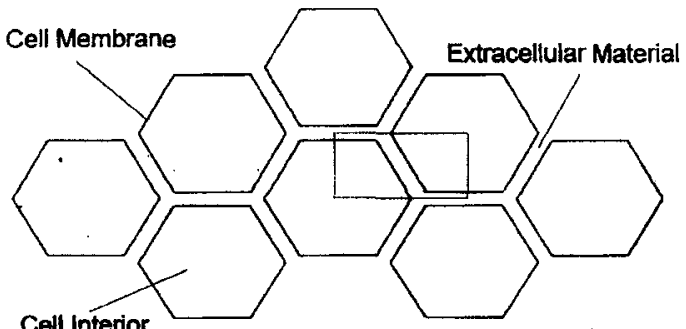

(b)

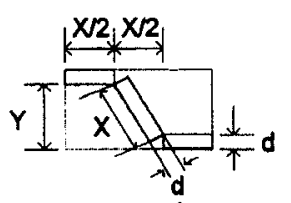

(a)

(c)

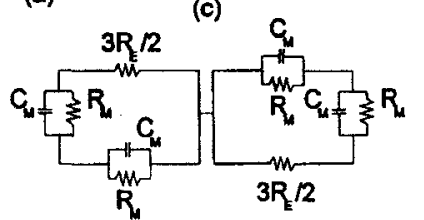

Figure 2. The conceptual and equivalent circuit models of the tissue conductivity in the tranverse direction to the muscle fibers. a) An illustration of group of muscle fibers with a box outlining the region on which the equivalent circuit model is based. b) An expanded view of the region on which the equivalent circuit is based showing the dimension of the important geometric parameters. c) The equivalent circuit model obtained from the region shown in (b).

The equivalent circuit for the transverse direction is shown in Fig. 2c. and it is based on the path that the current has through the extracellular medium represented by $3 R_{E} / 2$ and the parallel paths through the cell membranes represented by $R_{M}$ and $C_{M}$. In our equivalent circuit model, the cytoplasmic resistance in the parallel direction is assumed to be negligibly small but in a more rigorous approach, it could be included in series with the parallel membrane impedance. The assumed values of the geometric and electrical parameters were presented in Table 1, and the resultant equivalent circuit component values are shown in Table 3.

\begin{tabular}{|l|l|}
\hline$R_{E}$ & $3.741 \times 10^{4} \mathrm{Q}$ \\
\hline$R_{M}$ & $6.001 \times 10^{6} \mathrm{\Omega}$ \\
\hline$C_{M}$ & $1.250 \times 10^{-9} \mathrm{n}$ \\
\hline
\end{tabular}

Table 3. Circuit parameters for the transverse tissue equivalent circuit model.

The use of a distributed parameter equivalent circuit model in the transverse direction that incorporates spatial dependence does not yield results that are consistent with published measurements. Our results indicate that a single segment approach, as per Gielen [2], is adequate for simulating the conductivity in the transverse direction for a wide range of inter-electrode distances. 


\section{Results}

To calculate the effective conductivity from the boundary voltage and current of our equivalent circuit models we use the standard boundary definition of the effective conductivity as shown in (1).

$$
\sigma_{\text {eff }}=\frac{I d}{V S}
$$

In the effective conductivity equation $V$ represents the frequency dependent voltage phasor and $I$ represents the frequency dependent current phasor to which the voltage phase quantities are referenced. The area of the tissue perpendicular to current flow is represented by $S$ and $d$ is the distance between the electrodes.

The conductivity frequency response calculated from the equivalent circuit models are similar to those predicted by Gielen's [2] analytical model. Fig. 3. shows a comparison between the conductivity magnitude and phase predicted by the equivalent circuit modelling approach and that predicted by Gielen's [2] modelling approach.
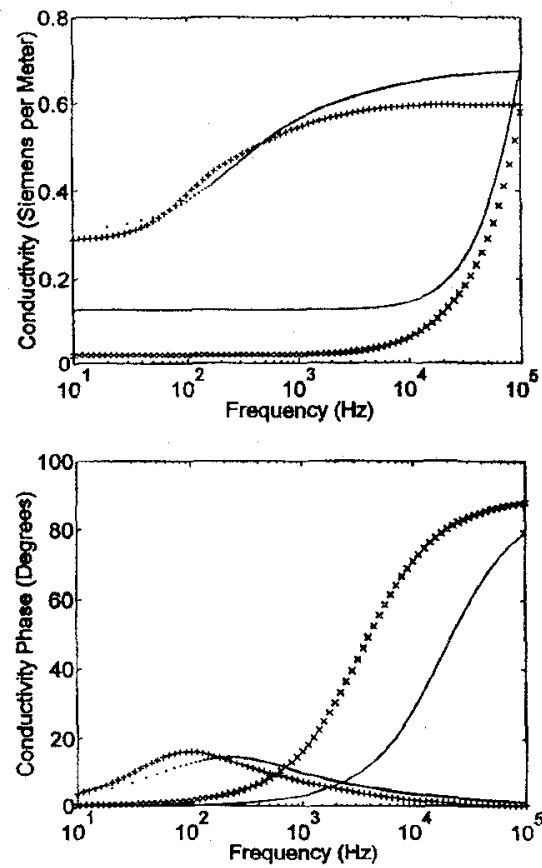

Figure 3. Graphs comparing the effective conductivity frequency response magnitude and phase predicted by the tissue equivalent circuit models and the analytical models proposed by Gielen et al. [2] for the electrical and geometric parameters given in Table 1, $X$ - Equivalent circuit simulation in the transverse direction. - - Gielen's transverse direction analytical formula. + Equivalent circuit simulation in the parallel direction. Gielen's parallel direction analytical formula.

The effective conductivity frequency response predicted by Gielen's analytical formula and that predicted by the equivalent circuit models for the direction parallel to the muscle fibers are in close agreement. It can be seen from Fig. 3. that the equivalent circuit model predicts a peak in the phase response at a slightily lower frequency than the analytical formula. The discrepancies between Gielen's formula and the equivalent circuit model effective conductivity frequency responses can be attributed to the finite length of each segment associated with the equivalent circuit modelling approach. In the transverse direction, Gielen's analytical formula predicts a marginally higher baseline for the low frequency conductivity magnitude as well as a larger low phase shift bandwidth than the equivalent circuit models.

\section{Discussion}

We have presented equivalent circuit models of the effective conductivity frequency response parallel and perpendicular to the direction of fibers in skeletal muscle tissue. These models are derived from the idealized physical geometry of the muscle fibers and the electrical parameters of the extracellular, cytoplasmic and cell membrane materials. A comparison between the conductivity frequency response of the equivalent circuit models and other models presented in the literature demonstrates a close agreement between the different modelling approaches. The equivalent circuit modelling approach offers several advantages over and above other approaches presented in the past. An equivalent circuit approach to tissue conductivity modelling is analogous to the physical situation that would be encountered in laboratory measurements of conductivity. Another problem with other modelling approaches is that they are not easily combined with electronic circuit models of biomedical instruments. The real versatility of the equivalent circuit model is in its ease of integration with circuit models for instruments that are used in conjunction with tissue. With these models electronic instruments, such as tissue stimulator circuits, can be simulated in combination with a circuit that simulates the frequency and spatial dependence of the tissue load thus facilitating optimization of the instrument design.

This research was supported by a grant from the Natural Sciences and Engineering Research Council.

\section{References}

P. W. Nicholson, "Specific impedance of cerebral white matter," Experimental Neurology vol. 13, pp. 386-401, 1965.

F. L. H. Gielen, H. E. P. Cruts, B. A. Albers, K. L. Boon, W. Wallinga-de Jonge and H. B. K. Boom, "Model of electrical conductivity of skeletal muscle based on tissue structure, "Medical \& Biological Engineering \& Computing vol. 24, pp. 34-40, 1986. R. Plonsey and R. Barr, "The four-electrode resistivity technique as applied to cardiac muscle, "IEEE Transactions on Biomedical Engineering vol. BME-29, no. 7, pp. 541-546, 1982. 